\title{
Anastigmatic Imaging with Unconstrained Object to Image Mapping
}

\author{
Juan C. Miñano , Jiayao Liu ,Pablo Benítez ，Milena Nikolic , Bharathwaj Narasimhan
}

\begin{abstract}
Anastigmatic imaging of an object to an image surfaces without the point-to-point mapping prescription and using a single optical surface is analyzed in 2D and 3D geometries (freeform and rotational-symmetric). Several design techniques are shown.
\end{abstract}

\section{Introduction}

With the aim to explore the relationship between number and size of the (refractive or reflective) surfaces used in an optical design with the maximum imaging quality achievable within the Geometrical Optics framework, we removed the object to image mapping constrain. In most imaging applications using optoelectronic devices, mapping between the sensor and the display can be modified by software and so prescribing a mapping between the object and the image of the optical system is unnecessary. There are exceptions, of course. For instance when the pixel sizes (at the sensor or at the display) imposes conditions on the maximum stretching or compressions in the mapping between object and image. We have also analyzed cases where not even the image plane is constrained but instead an image surface comes as a result of the design calculation when all the degrees of freedom are exhausted in getting sharp imaging of its points.

In a recent presentation [1] we analyzed several design strategies to get sharp imaging of multiple object points. Two conjectures arose from this analysis: 1) Let's define as optimum solution of an imaging design problem as the one providing the minimum average RMS spot diameter on a prescribed region of the image. The first conjecture establishes that if such optimum solution exists then it is "close" to a solution providing sharp imaging (i.e., zero RMS spot diameter) for a finite set of object points. This conjecture is important because the SMS design method provides a direct algorithm to this last solution and from it we can proceed to the optimum solution with a classical optimization technique. 2) The second conjecture is that the maximum number of object points $N$ that can be imaged by an optical system is given by this expression:

$$
N \leq \sum_{\text {optcal suffuces }} \frac{\text { surface area }}{\text { beam-print area }}
$$

For the preceding calculation a single ray bundle imaging an object point is considered (it is assumed that the result is approximately independent of the particular object point chosen). The bundle intercepts the different optical surfaces in its trajectory from the object to the image. The interception region at each optical surface is called beamprint area at this surface. The sum of the ratios of optical surface over the beam-print areas gives this upper bound. Obviously each term of the sum is greater than 1 . If this conjecture were true it will provide an easy way to check if an optical design problem is under or over-determined and should help to know (in a way that we don't know yet) if an optical system uses more optical surfaces that the minimum needed. From this point of view, the most interesting designs (those using the minimum number of surfaces) are those achieving equality in Eq. (1). Note also that a single surface can give an $N$ as big as desired, provided its area is bigger that $N$ times the beam-print area.

It happens that when a problem is such that the upper bound in Eq (1). is reached, then the mapping between the $N$ object points and their images is not free. For instance, this happens for the designs in ref [2] and [3]. For this reason we want to explore here imaging designs aiming to anastigmatic imaging (i.e. without astigmatism) for the entire field or for a finite number of object points in the field. In this work novel imaging designs with a single freeform optical surface (either refractive or reflective) are presented. In these designs, not only the mapping is obtained in the design process, but also the shape of the image surface is found. In the examples considered, the object is virtual and located at infinity and is seen from a pupil, which can emulate a human eye. In the first introductory part, we review $2 \mathrm{D}$ and rotational $3 \mathrm{D}$ designs obtained with the differential equation method for the limit case of small pupil. These rotational 3D designs don't have enough degrees of freedom to allow choosing a flat image surface and in general provide a curved image surface. In the second part the differential equation method for .the anastigmatic 3D rotational design (at the small pupil limit) is extended to 3D freeform design. The result of the 
extended method has been proved to coincide with the former 3D design by rotation which is a special case of 3D freeform design when the boundary conditions force that symmetry. The boundary condition can also be used to control the shape of the image surface, for instance to get a much flatter image surface than the one got with the 3D rotational design. Nevertheless there are not enough degrees of freedom to get complete flatness.

\section{2D design and 3D rotational design}

Figure 1 shows a refractive and a reflective design consisting of a single optical surface focusing the rays with angle $\theta$ respected to the optical axis to the image plane, at the image point $x(\theta)$. The designs can be obtained with different techniques: As a .Compound Cartesian oval design, as an SMS ([4].[5].[6].) design and as the solution of a second order ordinary differential equation ODE which, using the wavefront curvatures transformation equations (see for instance in [7].), establishes (in the small pupil approximation) that the rays must converge at the image plane. More detail of the calculations can be found in [8].
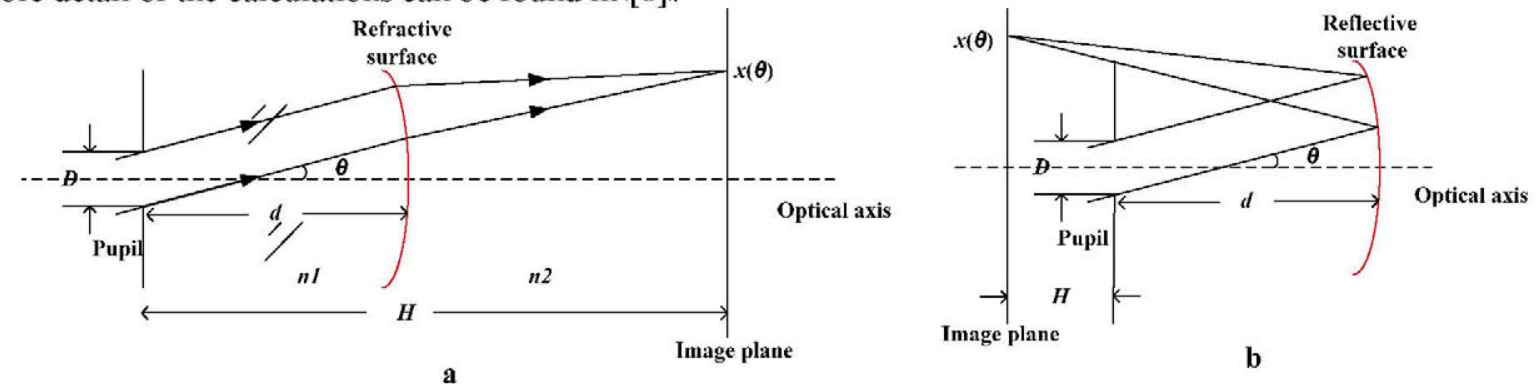

Figure 1. Sketch map of the refractive design (a) and the reflective design (b)
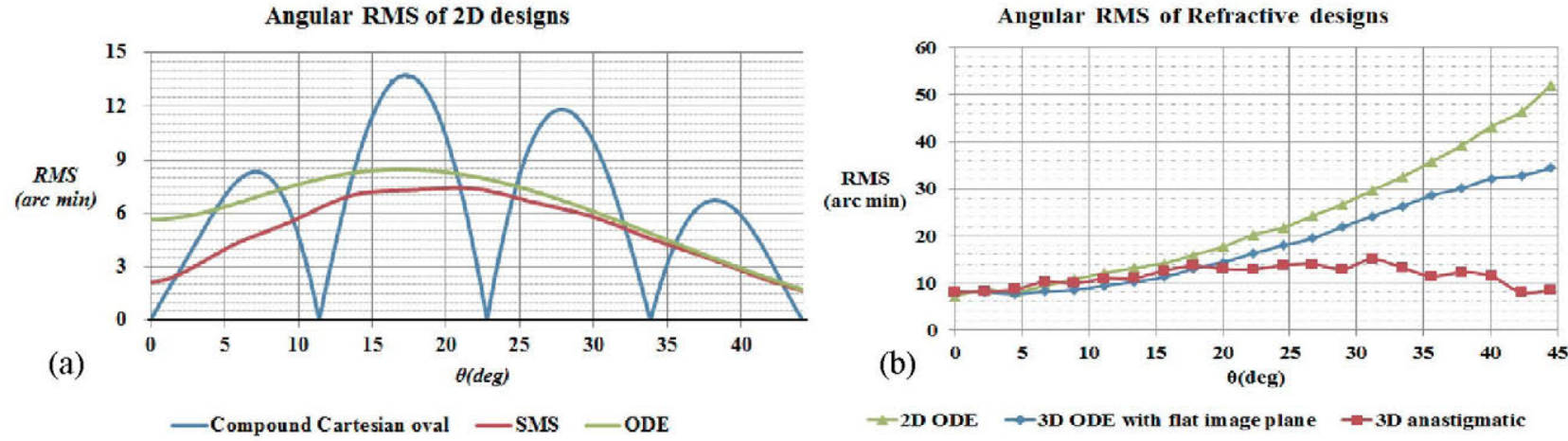

Figure 2. (a) RMS of the different 2D design techniques (b) RMS of the rotational 3D designs

Figure 2a shows the angular RMS of the 3 designs. The compound Cartesian oval works perfectly when the incident angle equals to the designing angle, but contains the highest RMS when far away from them, thus the curve shows a wavy shape. The SMS method considers two edge rays from the pupil for the imaging point, which has smoothed and improved the performance. The ODE method is based on the local properties of an infinitesimal part of the surfaces, which only deals with an infinitesimal bundle of rays, a single ray in other words, from point sources, thus provides little bit worse results than SMS method.

A 3D design can be obtained by rotating the former 2D designs on the optical axis. When doing this, not only the tangential condition, but also the sagittal condition has to be taken into account. Though SMS method has provided the best angular RMS result in 2D designs, when extended to 3D rotational cases, in the tangential plane, the rays follow the same condition in 2D and focus on the correspondent position on the rotated image plane, but this doesn't happen in the sagittal plane. We have several possible strategies. For instance, we can design a refractive surface that makes the middle distance between the tangential image surface and the sagittal image surface a flat image plane in order to get a uniform astigmatism at this plane. Its RMS is shown in Fig $2 \mathrm{~b}$ (blue curve). Or we can simply rotate the 2D ODE solution (green curve). Or we can design such that the tangential image surface and the sagittal image surface coincide to get anastigmatic design (see Fig. 3a). In this last case we cannot choose that this surface be a plane. Its RMS is shown in the red curve of Fig $2 b$. 

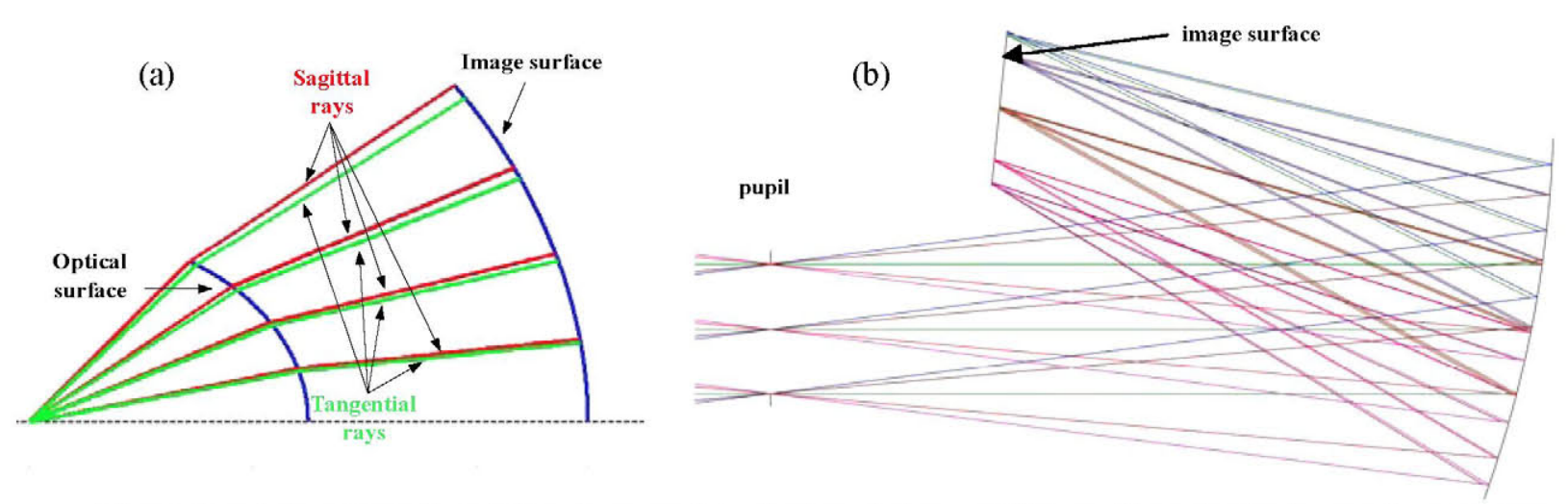

Figure 3. (a) Anastigmatic rotational symmetric dioptric design and (b) anastigmatic freeform mirror design

\section{3D free-form design}

A flat image plane is always much more desirable. We can apply the same the wavefront curvatures transformation equations to the 3D freeform anastigmatic design. In this general case we get a second order hyperbolic partial differential equation of which the rotational symmetric design is a particular solution when the boundary conditions are rotational symmetric. Fig 3 shows a particular design for a quite flat image surface althought the design does not have enough degrees of freedom to impose this surface to be flat. Only a curve on the image surface can be imposed.

\section{Conclusions}

We have analyzed anastigmatic designs consisting of a single surface in $2 \mathrm{D}$ and in $3 \mathrm{D}$ geometries. These last designs are in general freeform. The different 2D design techniques (Compound Cartesian oval, SMS and ODE) lead to identical designs at the small pupil limit. One of the design techniques (ODE) is based in a ordinary differential equation which is obtained when the wavefront curvatures transformation equations are applied to our particular problem. This design technique has been extended to the 3D case, where it provides two surfaces (deflecting surface and image surface) which in general are free-form. No prescribed mapping between the object and the image is imposed with the aim to use all the design degrees of freedom in getting the best anastigmatic design. The successful extension to 3D freeform designs has shown a very good result for applications.

\section{References}

[1] P. Benítez, J.C. Miñano, M. Nikolic, L. Jiayao, J.M. Infante, F. Duerr, "Sharp imaging of Multiple object pointS: How and Why"; Freeform Optics, Tucson, Arizona United States, November 3-7, 2013.

[2] Fabian Duerr, Pablo Benítez, Juan C. Miñano, Youri Meuret, and Hugo Thienpont, "Analytic free-form lens design in 3D: coupling three ray sets using two lens surfaces" Optics Express, Vol. 20, Issue 10, pp. $10839-10846$ (2012) http://dx.doi.org/10.1364/OE.20.010839.

[3] Fabian Duerr, Pablo Benítez, Juan C. Miñano, Youri Meuret, and Hugo Thienpont, "Analytic design method for optimal imaging: coupling three ray sets using two free-form lens profiles"; Optics Express, Vol. 20, Issue 5, pp. 5576-5585 (2012), http://dx.doi.org/10.1364/OE.20.005576.

[4] JC. Miñano, Pablo Benítez, Wang Lin, Fernando Muñoz, José Infante, Asunción Santamaría, "Overview of the SMS design method applied to imaging optics," Proc. SPIE 7429, Novel Optical Systems Design and Optimization XII, 74290C (2009)

[5] R. Winston, J. C. Miñano, P. Benitez, "Nonimaging Optics", Academic Press, New York, 181-217 (2005).

[6] J. Chaves, "Introduction to Nonimaging Optics" CRC Press, Boca Raton, FL, 271-324 (2008).

[7] O. N. Stavroudis, "The Mathematics of Geometrical and Physical Optics: The k-function and its Ramifications", Wiley-VCH, Berlin, 97-114 (2006).

[8] Jiayao Liu ; Juan C. Miñano ; Pablo Benítez and Lin Wang; "Single optical surface imaging designs with unconstrained object to image mapping", in Proc. SPIE 8550, Optical Systems Design 2012, 855011 (December 18, 2012); http://dx.doi.org/10.1117/12.981210. 